\title{
3 Shared Decision Making: Arzt und Patient entscheiden gemeinsam über die Behandlung
}

Christine Kuch

„Was kann ich tun, wenn Patienten sich einfach nicht an das halten, was ich verordnet habe?“ „Wie kann man Patienten dazu bringen, das zu tun, was gut für sie ist?“ „Kann ich als Arzt dafür verantwortlich gemacht werden, wenn mein Patient nicht „compliant“ ist?“

Wen solcherlei Fragen hin und wieder beschäftigen, kann mit dem Ansatz des Shared Decision-Making, zu Deutsch: partizipative Entscheidungsfindung, erfreuliche Erfahrungen machen. Zum Treffen einer optimalen Entscheidung sind demnach, neben dem medizinischen Wissen und der Behandlungserfahrung des Arztes, die individuellen Werte und Präferenzen des Patienten von zentraler Bedeutung. Ihre Berücksichtigung ist unerlässlich. Dies entspricht der zentralen Qualitätsmanagementstrategie, Prozesse an den Bedürfnissen der Kunden auszurichten. Im Rahmen der partizipativen Entscheidungsfindung werden die Bedürfnisse des Patienten direkt in den Behandlungsprozess integriert.

In diesem Kapitel wird das Konzept der partizipativen Entscheidungsfindung kurz erläutert und ihre Angemessenheit diskutiert. Die praktische Umsetzung im Alltag mit konkreten Vorgehensweisen für das Arzt-PatientenGespräch sowie Erfahrungen, die Praktiker mit der Anwendung des Konzepts gemacht haben, sind anhand von zwei Beispielen in Teil VI beschrieben (partizipative Entscheidungsfindung bei Kreuzschmerzen [s. Kap. VI.6] und bei Depression [s. Kap. VI.7]). 
Zur Erläuterung und Abgrenzung der partizipativen Entscheidungsfindung wurde ein hilfreiches Modell von Charles und Kollegen (1999) entwickelt. Sie differenzieren zwischen der partizipativen, der paternalistischen und der autonomen Entscheidung und gliedern den Entscheidungsprozess von Arzt und Patient in drei Phasen:

1. Informationsaustausch

2. Abwägen von Informationen

3. Treffen der Entscheidung

In einem partizipativen Entscheidungsprozess vermittelt der Arzt dem Patienten im Rahmen des Informationsaustauschs alle für die Entscheidung relevanten medizinischen Inhalte, während der Patient dem Arzt alle entscheidungsrelevanten persönlichen Informationen gibt. Demgegenüber erfolgt beispielsweise im Rahmen eines paternalistischen Entscheidungsprozesses der Informationsaustausch eher einseitig: der Arzt informiert den Patient, soweit er es für notwendig erachtet, über die Behandlungsalternativen. Das Abwägen der Informationen und das Treffen der Entscheidung erfolgt im partizipativen Entscheidungsprozess gemeinsam: Es geht hier nicht darum, dass der eine oder der andere „seinen Kopf“ durchsetzt, sondern dass sich die beiden Beteiligten ihre Überlegungen mitteilen und man auf diese Weise zu einer gemeinsamen Entscheidung kommt. Charles und Kollegen gehen davon aus, dass die unterschiedlichen Entscheidungsformen (partizipativ, paternalistisch oder autonom) alle innerhalb einer Konsultation auftreten können. Ihrer Ansicht nach ist es wichtig, dass der Arzt sein Verhalten den Bedürfnissen des Patienten anpasst und ihn insofern weder überfordert und alleine lässt, noch überprotegiert und bevormundet.

Die Angemessenheit und Umsetzung der partizipativen Entscheidungsfindung wird in der Literatur diskutiert. Wie ist Ihre Meinung dazu?

\section{Aufgabe 13 für anwendungsfreudige Leser}

Bitte schätzen Sie ein, inwieweit die folgenden Aussagen in Bezug auf die Teilhabe von Patienten an Behandlungsentscheidungen zutreffend sind oder nicht und tragen Sie dies in die Tabelle 12 ein.

\section{1. „Ärzte können bei ihren Patienten gut einschätzen, inwieweit sie sich an Entscheidungen beteiligen wollen."}

Man könnte darauf antworten: „Ich schon - wie das bei anderen aussieht, kann ich nicht wissen. " Man könnte jedoch auch davon ausgehen, dass auch Ärzte Menschen und damit nur eingeschränkt zur Hellseherei in der Lage sind. Und da wir wissen, dass Kommunikation immer mehrdeutig ist (Watzlawick 1969), wird es kaum möglich sein, ohne einen direkten verbalen Austausch mit dem Patienten zu „erfühlen“, wie der einzelne Patient dazu steht. 
Tab. 12 Einschätzung zutreffender Behandlungsentscheidungen

\begin{tabular}{|c|c|c|c|c|}
\hline & trifft zu & $\begin{array}{l}\text { trifft eher } \\
\text { zu }\end{array}$ & $\begin{array}{l}\text { trifft eher } \\
\text { nicht zu }\end{array}$ & $\begin{array}{l}\text { trifft nicht } \\
\mathrm{zu}\end{array}$ \\
\hline \multicolumn{5}{|l|}{$\begin{array}{l}\text { 1. „Ärzte können bei ihren Patienten gut } \\
\text { einschätzen, inwieweit sie sich an Entscheidun- } \\
\text { gen beteiligen wollen." }\end{array}$} \\
\hline \multicolumn{5}{|l|}{$\begin{array}{l}\text { 2. „Ärzte beteiligen ihre Patienten meistens } \\
\text { sowieso schon bei Entscheidungen." }\end{array}$} \\
\hline \multicolumn{5}{|l|}{$\begin{array}{l}\text { 3. „Ältere Menschen und Menschen mit } \\
\text { geringerem Bildungsstand wollen sich kaum an } \\
\text { den Entscheidungen beteiligten." }\end{array}$} \\
\hline \multicolumn{5}{|l|}{$\begin{array}{l}\text { 4. „Patienten können sich nur sehr eingeschränkt } \\
\text { an den Entscheidungen beteiligen.“ }\end{array}$} \\
\hline \multicolumn{5}{|l|}{$\begin{array}{l}\text { 5. „Patienten in Entscheidungen einzubeziehen ist } \\
\text { zwar eine schöne Idee, aber in der Alltagshektik } \\
\text { nicht machbar.“ }\end{array}$} \\
\hline $\begin{array}{l}\text { 6. „Patienten in Entscheidungen einzubeziehen ist } \\
\text { zwar eine schöne Idee, bringt aber letzten Endes } \\
\text { nichts.“ }\end{array}$ & & & & \\
\hline
\end{tabular}

Die Antwort auf die oben gestellte Frage könnte also lauten: „Ja, Ärzte können das sogar sehr gut einschätzen, wenn sie ihre Patienten über die Behandlungsalternativen mit ihren unterschiedlichen Vor- und Nachteilen informiert haben und sie anschließend fragen, ob sie sich an der Entscheidung beteiligen wollen."

Da Ärzte wohl häufig auf diesen Austausch verzichten, sind die Ergebnisse in Studien ernüchternd: Ärzte unterschätzen oft, inwieweit ihre Patienten informiert und an Entscheidungen beteiligt werden wollen (u.a. Coulter et al. 1997; Entwhistle et al. 1998, Floer et al. 2004) und wissen häufig wenig von der Meinung und den Erwartungen der Patienten in Bezug auf die Behandlung (Cockburn et al. 1997; Rothenbacher et al. 1997, Britten 2004).

\section{2. „Ärzte beteiligen ihre Patienten meistens sowieso schon bei Entscheidungen.“}

Die Mehrheit der Patienten gibt vielen Studien zufolge an, an Entscheidungen beteiligt werden zu wollen, jedoch nur ein kleiner Teil davon sieht diesen Wunsch realisiert. (z. B. Coulter et al. 2003, Dierks et al. 2005). Das bedeutet jedoch nicht, dass Ärzte nicht den Versuch machen, Patienten bei Entscheidungen einzubeziehen. Allerdings scheint der Versuch bei einem Großteil der Patienten zu misslingen. Grundsätzlich zeigen Studien, dass Ärzte dem Konzept der partizipativen Entscheidungsfindung mehrheitlich positiv gegenüberstehen (z. B. Floer et al. 2004, Charles et al. 2004). Auch die kanadische Wissenschaftlerin O'Connor und ihrer Kollegen konstatieren eine deutliche 
Zunahme an Leitlinien, in denen empfohlen wird sicherzustellen, dass Patienten ihre Behandlungsoptionen mit den entsprechenden Vor- und Nachteilen kennen und Patienten zu helfen, eine Entscheidung entsprechend der persönlichen Werte und Bedingungen zu treffen (O'Connor et al. 2002). Ein Beispiel aus Deutschland ist die „Hausärztliche Leitlinie zur hausärztlichen Gesprächsführung " der hessischen Leitliniengruppe (s. Linksammlung).

\section{3. „Ältere Menschen und Menschen mit geringerem Bildungsstand wollen sich kaum an den Entscheidungen beteiligten."}

Diese Einschätzung wird teilweise von Studienergebnissen unterstützt, wobei darüber hinaus auch die folgenden Faktoren positiv mit der Präferenz für eine Entscheidungsbeteiligung zusammenhängen: „weibliches Geschlecht“, „für die Behandlungsentscheidung relevante Informationen erhalten zu haben“ und „eher aktive Coping-Strategien der Person“. Regressionsmodelle zeigen jedoch eine nur geringe Aufklärung der Varianz durch soziodemographische Variabeln wie Alter, Geschlecht und Bildung (Degner et al. 1992, Beaver et al. 1996, Ende et al. 1989, Adams et al. 2001). Das bedeutet, dass diese Variablen zwar eine Rolle spielen, aber nur von geringer Bedeutung sind für die sich schließlich in einer bestimmten Situation ergebende Präferenz.

Es wäre also schlicht falsch anzunehmen, ein alter Patient wolle sich nicht beteiligen. Das Zustandekommen der Beteiligungspräferenz ist zu komplex, als dass man es auf Alter oder Bildungsstatus reduzieren könnte. Eine größere Rolle scheinen zudem interaktionsbezogene Variablen, wie z. B. der Claube daran, dass der Arzt einen überhaupt einbeziehen möchte, oder das Vertrauen, das man in seinen Arzt hat, zu spielen (Kraetschmer et al. 2004, Dierks et al. 2005).

\section{4. "Patienten können sich nur sehr eingeschränkt an den Entscheidungen beteiligen."}

Auf Patienten, die als Notfall ins Krankenhaus eingeliefert werden, auf schwer demente Patienten oder auf Patienten, die gerade von einer lebensbedrohlichen Diagnose erfahren haben, wird dieser Satz wohl zutreffen. Zugleich zeigen eine Reihe von Studien, dass beispielsweise auch Patienten mit psychiatrischen Erkrankungen an Entscheidungen teilhaben wollen (u. a. Hamann et al. 2005) bzw. dass es darauf ankommt, den richtigen Moment abzupassen, damit Patienten in der Lage sind, sich am Entscheidungsprozess zu beteiligen (u. a. Pereira 2000).

\section{5. „Patienten in Entscheidungen einzubeziehen ist zwar eine schöne Idee, aber in der Alltagshektik nicht machbar."}

Der britische Allgemeinmediziner Glyn Elwyn und Kollegen formulieren hierzu, dass selbstverständlich Zeitmangel eine Barriere zur Einbeziehung von Patienten in Entscheidungen sei, zumindest bei nicht trainierten Ärzten. Zu- 
gleich seien aber Ärzte nicht so leicht bereit zuzugeben, dass auch eine mangelnde Fähigkeit zur Umsetzung einer gemeinsamen Entscheidung ein wichtiges Hindernis ist. Elwyn und Kollegen weisen darauf hin, dass viele Ärzte nicht mit den Techniken vertraut sind, die für eine gemeinsame Entscheidungsfindung erforderlich sind (Elwyn et al. 1999). Entsprechend konstatieren auch Loh und Kollegen, dass Schulung von Ärzten sowie unterstützende Informationsmaterialen notwendige Bedingungen sind, damit SDM auch unter zeitlichen Aspekten machbar ist (Loh 2007).

\section{6. „Patienten in Entscheidungen einzubeziehen ist zwar eine schöne Idee, bringt aber letzten Endes nichts."}

Auf diese Frage wird an dieser Stelle nicht eingegangen - eine Fülle von Informationen hierzu sind in den Beiträgen von Andreas Loh und Sabine Beck in Kapitel VI.6 und VI.7 zu finden.

Letztlich gilt in Zusammenhang mit der partizipativen Entscheidungsfindung, dass ein wirksames Medikament nur wirksam werden kann, wenn der Patient es entsprechend der Anweisungen einnimmt. Eine Veränderung bei teilweise verhaltensabhängigen Erkrankungen wie z. B. Diabetes oder Bluthochdruck wird nur dann erfolgen, wenn der Patient auch tatsächlich sein Verhalten reguliert. Die Zufriedenheit mit einem Behandlungsergebnis wird sich nur dann einstellen, wenn die entsprechenden Erwartungen an die bestehenden Möglichkeiten adjustiert werden. Neben ethischen und nutzenökonomischen Überlegungen sind es somit auch rein am medizinischen Ergebnis orientierte Überlegungen, die die partizipative Entscheidungsfindung als potenziell vorteilhaft erscheinen lassen.

\section{Literatur}

Adams RJ, Smith B], Ruffin RE: Patient preferences for autonomy in decision making in asthma management. Thorax 2001; 56(2): 126-132

Beaver K, Luker KA, Owens RG, Leinster SI, Degner LF, Sloan JA: Treatment decision making in women newly diagnosed with breast cancer. Cancer Nurs 1996; 19(1): 8-9

Britten N: Patients' expectations of consultations. Patient pressure may be stronger in the doctor's mind than in the patient's. BMJ 2004; Feb 21; 328(7437): 416-7

Charles C, Gafni A, Whelan T: Self-reported use of shared decision-making among breast cancer specialists and perceived barriers and facilitators to implementing this approach. Health Expectations 2004; 7(4): 338-348

Charles C, Gafni A, Whelan T: Decision-making in the physician-patient encounter: revisiting the shared treatment decision-making model. Soc Sci Med 1999; 49: 651-661

Cockburn I, Pit S: Prescribing behaviour in clinical practice: patients' expectations and doctors' perceptions of patients' expectations - a questionnaire study. BMI 1997; Aug 30; 315(7107): 520-3

Coulter A, Magee $\mathrm{H}$ : The European patient of the future. Berkshire: Open University Press 2003

Coulter A: Partnerships with patients: the pros and cons of shared clinical decision-making. I Health Serv Res Policy 1997; 2(2): 112-121

Degner LF and Sloan JA: Decision making during serious illness: what role do patients really want to play? I Clin Epidemiol 1992; 45(9): 941-950 
Dierks ML, Seidel G: Gleichberechtigte Beziehungsgestaltung zwischen Ärzten und Patienten - wollen Patienten wirklich Partner sein? In Härter M, Loh A, Spies C (Hrsg.): Gemeinsam entscheiden- erfolgreich behandeln. Köln: Deutscher Ärzte-Verlag 2005

Elwyn G, Edwards A, Kinnersley P: Shared decision-making in primary care: the neglected second half of the consultation. Br / Gen Pract 1999; 49(443): 477-82

Ende J, Kazis L, Ash A, Moskowitz MA: Measuring patients' desire for autonomy: decision making and information seeking preferences among medical patients. I Gen Intern Med 1989; 4(1): 23-30

Entwistle VA, Sheldon TA, Sowden A, Watt IS: Evidence-informed patient choice: Practical issues of involving patients in decisions about health care technologies. International Journal of Technology Assessment in Healthcare 1998; 14(2): 212-225

Floer B, Schnee M, Böcken I, Streich W, Kunstmann W, Isfort J, Butzlaff M: Shared Decision Making. Gemeinsame Entscheidungsfindung aus der ärztlichen Perspektive. Medizinische Klinik 2004; 99(8): 435-440

Hamann I, Cohen R, Leucht S, Busch R, Kissling W. Do patients with schizophrenia wish to be involved in decisions about their medical treatment? Am J Psychiatry 2005; 162: 2382-4

Kraetschmer N, Sharpe N, Urowitz S and Deber RB: How does trust affect patient preferences for participation in decision-making? Health Expectations 2004; 7(4): 317-326

Loh A, Simon D, Kriston L, Härter M: Patientenbeteiligung bei medizinischen Entscheidungen. Effekte der Partizipativen Entscheidungsfindung aus systematischen Reviews. Deutsches Ärzteblatt 2007; 104 (21): 1483-8

0'Connor AM, Jacobsen M], Stacey D: An Evidence-Based Approach to Managing Women's Decisional Conflict. Journal of Obstetric, Gynecologic, and Neonatal Nursing 2002; 31(5): 570-581

Pereira B|: Optimization of pre-ESRD care: the key to improved dialysis outcomes. Kidney Int 2000; 57(1): 351-365

Rohenbacher D, Lutz MP, Porzsolt F: Treatment decisions in palliative cancer care: Patients' preferences for involvement and doctors' knowledge about it. European Journal of Cancer 1997; 33(8): 1184-9

Watzlawick P, Beavin JH, Jackson D: Menschliche Kommunikation: Formen, Störungen, Paradoxien. 11. Aufl. Bern: Huber 2007

\section{links}

Gesprächsführungs-Leitlinie der KV Hessen

http://www.leitlinien.de/leitlinienanbieter/deutsch/pdf/hessengespraech

Informationen zur Partizipativen Entscheidungsfindung

www.patient-als-partner.de

Sammlung englischsprachiger, geprüfter Entscheidungshilfen

http://decisionaid.ohri.ca/index.html

Beispiel für eine deutsche, geprüfte Entscheidungshilfe der Uni Hamburg

http://www.mammographie-screening-online.de/Mammographie_Online/mammographie_online.html

... und weitere geprüfte Patienteninformationen der Uni Hamburg

http://www.gesundheit.uni-hamburg.de/cgi-bin/newsite/index.php?page=page_167

... sowie des IQWIG

http://www.gesundheitsinformation.de/index.de.html 